\title{
Mist begins to clear for lung delivery of RNA
}

\author{
Inhaled RNA is coming soon for countermeasures against respiratory pathogens, including COVID-19.
}

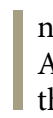

n August, Vir Biotechnology and

Alnylam Pharmaceuticals announced

that lung-targeted small interfering RNA (siRNA) conjugates against SARS-CoV-2 and other coronaviruses delivered to the lung are scheduled for preclinical studies by the end of the year. The collaboration inked in March involves Alnylam, the RNA interference (RNAi) pioneer, providing Vir with over 350 siRNAs targeting all available SARS-CoV-1 and SARS-CoV-2 genomes. Vir has been screening these molecules in vitro for potent lead siRNA candidates; if any are taken forward, Alnylam retains a 50-50 option for participation.

Around the same time, Translate Bio closed a licensing deal with French pharmaceutical giant Sanofi Pasteur for use of its mRNA platform to develop vaccines for infectious diseases. The deal provides $\$ 425$ million up front and up to $\$ 1.9$ billion in milestones and royalties on worldwide sales. The new agreement expands on a 2018 deal covering five infectious diseases and a March 2020 update adding COVID-19 to the list of vaccine targets.

Under the partnership, the mRNA vaccines will be delivered by intramuscular injection and are not targeted to specific organs or tissues. But the multibillion-dollar deal highlights the potential of Translate Bio's expertise in mRNA delivery. That includes a tissue-specific mRNA delivery platform that the company is using to target the lung in cystic fibrosis and idiopathic pulmonary fibrosis. RNA delivery into the lungs, if successful, would be a boon for drug and vaccine makers. Other advances in lipid nanoparticle (LNP) formulations, inhalation devices, carrier particles and customized chemical modifications are making strides toward the goal of delivering RNA candidates - including mRNA, antisense RNA and siRNA - into the lung. Success could open treatment doors for lung disorders in COVID-19 and beyond.

Platforms that deliver to the lung are attractive for two main reasons. First, they offer direct access to the alveoli and lung parenchyma for treating disorders, such as asthma, idiopathic pulmonary fibrosis, lung cancer and a range of pulmonary infections. Second, they are not only non-invasive, they also offer hundreds of square meters of well-perfused surface area for rapid drug absorption and avoid alteration by enzymes, as occurs in the gut and liver during oral delivery. If researchers

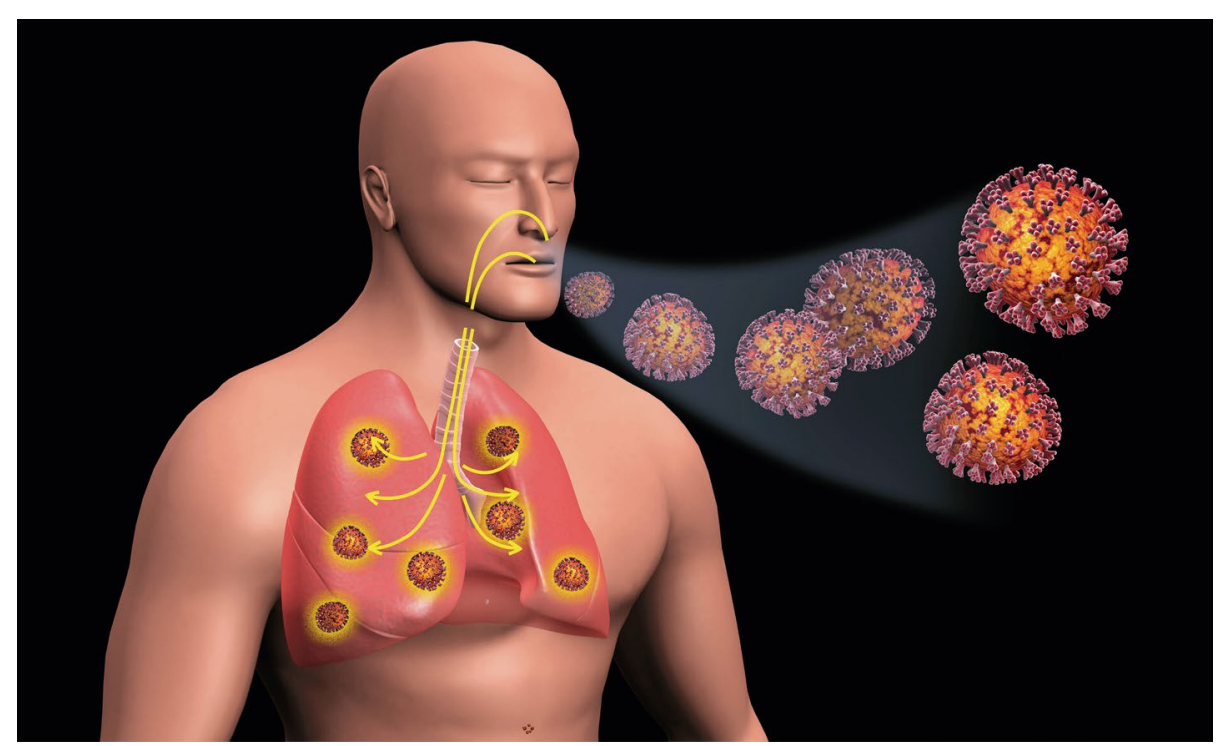

SARS-CoV-2 infects lung tissue through breathing; drug makers could use the same route to deliver RNA treatment. Credit: Stocktrek Images / Science Source

can overcome the challenges of delivery to the lung, RNA-based therapeutics offer many advantages, such as the ability to directly knock down a viral infection, and convenient dosing without the need for an injection or infusion.

For small molecules such as glucocorticoids, inhalation has been successfully deployed for some time. Until recently, however, lung delivery for biologics and nucleic acids has remained largely unexplored. Some of the first attempts with inhaled insulin were a commercial failure. Pfizer's inhaled powder insulin product, Exubera, was approved in 2006 but was removed from the market just a year later owing to an unwieldy delivery device, marketing missteps, a high rate of adverse effects and a lung cancer scare. Similarly, disappointing sales led Sanofi to hand back rights to another powdered insulin product, Afrezza, to its developer MannKind less than two years after approval. Although Afrezza launched with a much more convenient inhaler, it has faced difficulties with insurance coverage, a 'black box' warning for bronchospasm and lingering concerns about lung cancer.

To deliver nucleic acids, including RNA-based therapeutics to the lung, several companies have pursued LNPs. A nanoparticle encapsulates and protects the mRNA from degradation and facilitates cellular uptake. Once inside the cell, the mRNA is released into the cytoplasm, where the cellular machinery takes over, ensuring proper folding and glycosylation of the translated protein. Nanoparticle delivery systems have the advantage that, once they are optimized for an initial target, the next product can have a greatly expedited timeline from preclinical proof of concept to clinical trials.

The first FDA-approved siRNA therapeutic, Alnylam's Onpattro for transthyretin amyloidosis, is packaged in a cationic amino MC3 lipid nanoparticle that targets hepatocytes. The LNP has been a workhorse for liver-specific therapeutic delivery and is featured in development programs at a number of other companies, including Silence Therapeutics and Moderna. Although Alnylam has moved away from LNP delivery to $\mathrm{N}$-acetylgalactosamine (GalNAc)-conjugated molecules, some other companies developing RNA-based therapeutics, such as Translate Bio, are leaning into this delivery platform because it can be targeted to different organs by varying the lipid composition of LNPs.

A LNP typically has up to four components, according to James Dahlman, a biomedical engineer with the Georgia Institute of Technology. These are an ionizable lipid, a cholesterol, a polyethylene 
glycol-conjugated lipid, and a phospholipid or other helper lipid. Dahlman has used DNA barcoding to rapidly screen hundreds of LNPs loaded with the mRNA Cre-lox system for distribution to cells outside the liver. What he learned from this and other experiments is that variations in those four particle components can determine delivery to specific cells. "It's a giant chemical space. All four of those variables matter. And there's dozens to hundreds of thousands of variants in each one of those variables," says Dahlman. The variables for optimized delivery can change depending on whether you're delivering from the circulation side, like Alnylam, or the airway side, as with Translate Bio's phase $1 / 2$ cystic fibrosis therapy. Dahlman is the cofounder of a company, Guide Therapeutics, developing nanoparticles based on this technology.

\section{"For the lungs specifically,}

\section{it's really about getting}

\section{good tissue penetration and} getting into the deep lung, because we're dealing with a hyper-branched organ and you

\section{want to coat the entire lung}

\section{surface."}

Scientists at the Massachusetts Institute of Technology have worked out how to tweak LNPs to suit lung delivery of mRNA therapeutics. In a study published in 2019 in Advanced Materials and partially funded by Translate Bio, they report a nanoparticle formulation that successfully delivers a firefly luciferase to the lung in mice using an inhaled nanoparticle containing hyperbranched poly(beta amino esters). Translate Bio has patents on cationic ionizable lipids.

The mechanical method for administering an inhaled drug is another challenge for developing RNA-based drugs. When it comes to pulmonary delivery, says Frank DeRosa, chief technology officer for Translate Bio, "it's really creating the ability to make your lipid nanoparticle aerosolize or fly, basically, or be able to be breathed in." Translate Bio is putting that principle to work in a phase $1 / 2$ cystic fibrosis clinical trial using its LNP platform to deliver an mRNA product to the lung through nebulization. DeRosa says maintaining integrity of the mRNA and its ability to penetrate the mucus layer of the lung tissue is critical to successful delivery. "We want lipids that are capable of passing through the mucus, getting into the cells and releasing the mRNA cargo. But at the same time, they need to be metabolized. They need to be cleared and not have side effects that you don't want," says DeRosa.

Dry powders are emerging as an alternative to aerosol delivery for inhalation. Acorda Therapeutics has a delivery platform based on dry powder inhalation. Acorda CEO Ron Cohen says their approach is to engineer powdered granules that resemble Wiffle balls. "There's a lot of surface area because of all of the curves, so even a slight breeze, a slight breath is going to spin it. Just a regular, easy inhalation can get large amounts of the drug in." Acorda's pipeline does not include any nucleic acid therapeutics - it markets Inbrija, a levodopa inhalation powder for Parkinson's disease - but the company's chief medical officer, Burkhard Blank, says the technical aspects of designing a dry powder formulation should be similar. Blank says that Acorda had the opportunity to work on a messenger RNA program for a federal agency for an undisclosed virus that was not COVID-19. That mRNA product encoded antibodies against the virus. Blank says, "it was demonstrated that there was indeed in the lung the expression of antibodies."

Vir and Alnylam will be taking a different approach to deliver their inhaled COVID-19 siRNA therapeutic into the lung. The product, ALN-COV, targets conserved regions shared by SARS-CoV and SARS-CoV-2, in what the company hopes will be an effective treatment for COVID-19 and potential future pandemic coronaviruses. According to Akin Akinc, general manager at Alnylam for Givlaari (givosiran), a subcutaneously administered RNAi approved by the US Food and Drug Administration for acute hepatic porphyria, Alnylam's delivery strategy for ALN-COV is informed by its experience with its discontinued respiratory syncytial virus candidate, which was formulated as a naked siRNA in saline. For ALN-COV, "we're using chemical modifications that enhance stability, prevent immunostimulation, and enhance the potency and durability of the pharmacological effect [of the siRNA]," says Akinc. In addition to the chemical modifications, Alnylam is using a ligand to facilitate distribution of the siRNA and uptake by lung cells. In June 2019, at the 3rd International Conference on the Long and the Short of Non-Coding RNAs in Chania, Greece, company scientists presented mouse data in which siRNAs delivered to the lungs as aerosols via a pressurized syringe showed similar potency and duration of activity to those of GalNAc-conjugated siRNA molecules delivered subcutaneously and targeted to the liver. The modified siRNA

\section{First oral SMA drug}

The US Food and Drug Administration has approved an oral drug for treating spinal muscular atrophy (SMA), developed by PTC Therapeutics and Roche's Genentech unit. Evrysdi (risdiplam) received a green light from the regulator on August 7 to treat patients aged 2 months and older. The small-molecule drug is a splicing modifier designed to boost full-length motor neuron 2 (SMN2) mRNA expression to increase functional SMN protein production in muscle. In SMA, low levels of SMN protein lead to motor neuron death and muscle atrophy. In trials, Evrysdi improved motor performance and increased survival in patients with SMA. It can be taken orally, which is an important option for patients with this rare and often fatal disease.

Evrysdi sits alongside two other approved SMA treatments: the antisense therapy Spinraza (nusinersen) from Biogen and Ionis Pharmaceuticals, which also targets SMN2 pre-mRNA, and Novartis's SMN1 gene therapy Zolgensma (onasemnogene abeparvovec). Evrysdi is an orally administered liquid, which gives patients the option of taking the daily dose at home, unlike Spinraza, which must be injected three times a year into the spine, and Zolgensma, a one-shot intravenous infusion (albeit one with only around 5 years of data available). At \$2.1 million, Zolgensma is also the world's most expensive drug, and Spinraza costs $\$ 750,000$ for the first year and $\$ 375,000$ every subsequent year. Evrysdi's lower price - a maximum of $\$ 340,000$ per year, scaled according to body weight - might afford it a competitive advantage. In addition, oral dosing achieves systemic exposure, which targets tissues and organs outside the central nervous system, so Evrysdi could potentially have a greater disease-modifying effect than Spinraza (although such benefit has yet to be proven clinically). Both Evrysdi and Spinraza can be used to treat children of all ages and adults, but Zolgensma only to treat children younger than two. Although Evrysdi is likely to be a serious competitor to Spinraza, not so the gene therapy Zolgensma, which will probably remain the treatment of choice for children under two years of age.

Published online: 5 October 2020 https://doi.org/10.1038/s41587-020-0706-x 
is used with a nebulizer to create particle size distributions that can access the deep lung as well as the upper airway. "For the lungs specifically, it's really about getting good tissue penetration and getting into the deep lung, because we're dealing with a hyper-branched organ and you want to coat the entire lung surface," says Akinc.

Ionis Pharmaceuticals is pursuing a similar strategy to improve the potency of its antisense oligonucleotide candidate targeting the epithelial sodium channel $(\mathrm{ENaC})$ in cystic fibrosis. Shuling Guo, vice president for antisense drug discovery at Ionis, says that the naked oligonucleotide is distributed well in the lung with aerosol delivery. A preclinical study has shown effective delivery of a 3-10-3 gapmer with three constrained ethyl residues at each terminus to the lung. But she calls GalNAc delivery "revolutionary" for oligonucleotides delivered to the liver. "So what we are exploring is to find a better kind of ligand-assisted delivery so that we can really have a much more potent compound going forward," says Guo.

Because these lung-targeted RNA-based therapeutics are still in early stages of development, the safety picture is not yet clear. Hasina Outtz Reed is a Weill Cornell Medicine pulmonary critical care physician and molecular and vascular biologist working on mouse models of lung injury. She's partnering with Sloan Kettering Institute chemist
Daniel Heller to engineer nanoparticles to deliver therapeutics to the lungs. Outtz Reed emphasizes that in vivo models will be needed to evaluate the new generation of lung-targeted therapeutics: "I think, as with anything, we need to make sure that we know where these particles end up. Anything you inhale there's theoretically going to be a high concentration, and the lungs are prone to having inflammatory reactions, so that's something else we have to keep an eye on."

\section{Catherine Shaffer}

Ann Arbor, MI, USA

Published online: 5 October 2020

https://doi.org/10.1038/s41587-020-0692-z

\section{Around the world in a month}

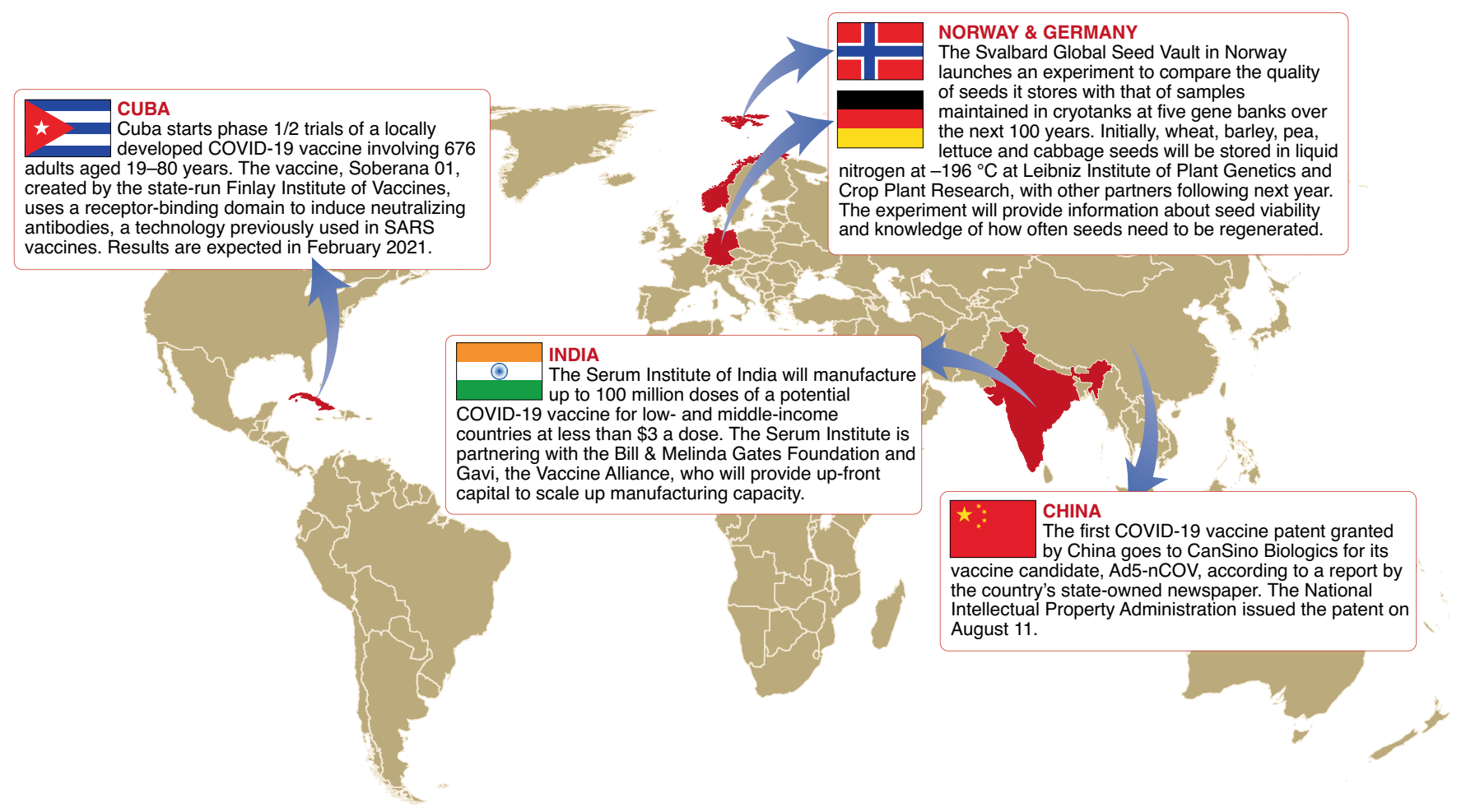

Credit: Map: (c) iStockphoto; Flags: pop_jop / DigitalVision Vectors / Getty

Published online: 5 October 2020

https://doi.org/10.1038/s41587-020-0691-0 